\title{
Binding-Time Annotations without Binding-Time Analysis
}

\author{
Wim Vanhoof and Maurice Bruynooghe \\ Department of Computer Science, K.U.Leuven, Belgium \\ e-mail: $\{$ wimvh, maurice\}@cs.kuleuven.ac.be
}

\begin{abstract}
The basic task of binding-time analysis (BTA) is to compute annotations that guide the unfolding decisions of a specialiser. The main problem is to guarantee that the specialisation will terminate. In the context of logic programming, only few automatic such analyses have been developed, the most sophisticated among them relying on the result of a separate termination analysis. In this work, we devise an analysis that generates the annotations during termination analysis, which allows much more liberal unfoldings than earlier approaches.
\end{abstract}

\section{Introduction}

Partial evaluation is a well-studied source-to-source transformation, capable of specialising a program $P$ with respect to a part $s$ of its input. The result is a program $P_{s}$ that computes, when provided with the remaining part $d$ of the input, the same result as the original program $P$ on the complete input $s$ and $d$. The general effect of partial evaluation is that the computations performed by a program are staged: some (ideally all) operations in $P$ that depend only on $s$ are performed by the specialiser; the remaining computations (those depending on $d$ ) by the residual program $P_{s}$. Partial evaluation can be used to speed up the computation of a program, in particular when the program must be run a number of times while part of its input (the part denoted by $s$ ) remains constant. Indeed, using partial evaluation, the computations depending on $s$ need to be performed only once to construct $P_{s}$, which can then be run any number of times with different inputs $d$. The heart of any partial evaluator is an evaluation mechanism for the language under consideration. In a logic programming setting, "evaluation" of a program corresponds to building an SLD-tree for a program/query pair $\langle P, Q\rangle$. If the program terminates, the corresponding SLDtree is finite. In this setting, partially available input corresponds to a query $Q^{\prime}$ that is less instantiated than $Q$. Due to the nature of logic programming, the program could, in principle, simply be evaluated with respect to $Q^{\prime}$. Most likely, however, the SLD-tree built for $\left\langle P, Q^{\prime}\right\rangle$ will be infinite. Indeed, if the control flow is determined by a value that is unknown in $\left\langle P, Q^{\prime}\right\rangle$, SLD-derivations of infinite length may be created resulting in a non-terminating specialisation process. Instead of building such a possibly infinite SLD-tree, a partial evaluator for logic programs builds a finite number of finite SLD-trees that together cover the 
complete computation for $\left\langle P, Q^{\prime}\right\rangle[14]$. The resulting SLD-trees are partial, in the sense that, while building the SLD-tree, the partial evaluator unfolds some predicate calls whereas it does not unfold others. The predicate calls that are not unfolded are said to be residualised - they will appear as code in the residual program.

Most work on partial evaluation in logic programming concentrates on the so-called on-line approach [11]: during the construction of a partial SLD-tree, the partial evaluator selects each call occurring in an SLD-derivation and decides whether or not to unfold it; usually basing its decision on the structure of the SLD-tree built so far. In the off-line approach on the other hand, the program is first analysed by a so-called binding-time analysis (BTA). Binding-time analysis is a global analysis that takes a program and (an abstraction of) the query and generates an annotated version of the original program, in which every predicate call is accompanied by an instruction stating whether or not instances of this call must be unfolded. The actual specialiser builds the partial SLD-trees simply by following the instructions generated by BTA. While in general an on-line partial deduction system can achieve better results than an off-line system, the off-line approach also offers a number of advantages. First of all, the separation of the process in a binding-time analysis followed by a specialisation phase makes the process conceptually easier to reason about, and results in a fairly simple (and efficient!) specialiser from which the burden of continuously monitoring the evaluation process has been removed. Also, the analysis output can be represented by annotations on the original source program, and provides as such excellent feedback to the user providing clues to why an optimisation was (not) performed. In spite of these advantages, only few efforts have been made to construct an off-line partial evaluator for logic programming, in particular [16] and [9]. Both approaches require, however, the binding-time analysis to be performed by hand. In previous work [18], we have developed a binding-time analysis for the strongly moded logic programming language Mercury. Adapting such an analysis to an unmoded language is far from trivial. To the best of our knowledge, the first serious attempt to create an automatic binding-time analysis for pure logic programs is [3]. The analysis advocates the use of termination conditions to decide what predicate calls can safely be unfolded. These conditions must, however, be created by hand or be derived by a separate termination analysis which imposes some serious restrictions on the unfolding possibilities as we will demonstrate further on. In this work, we generate the necessary annotations during termination analysis, which will allow for much more liberal unfoldings during specialisation.

The remainder of this paper is organised as follows: in Section 2 we motivate our work by demonstrating the need for a more refined control mechanism than the use of termination conditions alone. In Section 3 we adapt an existing termination analysis to our needs and develop the actual binding-time analysis. Section 4 reports on a prototype implementation of the analysis and we conclude in Section 5. 


\section{The Role of Termination in Binding-time Analysis}

The basic task of binding-time analysis is to annotate every predicate call in a program as either static or dynamic such that constructing an SLD-derivation by unfolding the statically annotated calls - leaving the dynamically annotated ones as they are - terminates for every call that may occur during specialisation. As was suggested before in the literature [3,7], it seems natural to base the decision whether or not to unfold a predicate call on the termination properties of the particular call. The motivation is obvious: if it can be shown that the (specialisation-time instance of the) call terminates under normal evaluation, the call can safely be annotated as static, since unfolding it during specialisation will terminate. First, we take a closer look at how the termination properties of a call can be expressed; next, we discuss the use of such properties for binding-time analysis.

The condition under which a call is guaranteed to terminate is usually expressed in terms of the degree of instantiatedness of the call's arguments, measured with respect to a given norm. Such a norm is a function that maps a term to an expression that approximates the "size" of the term. Two examples of frequently used norms are the termsize norm, denoted by $\|.\|_{t s}$, which counts the number of functors in a term, and the listlength norm, denoted by $\|.\|_{l l}$ which counts the number of elements in a list.

$$
\|t\|_{t s}=\left\{\begin{array}{ll}
1+\sum_{i=1}^{n}\left\|t_{i}\right\|_{t s} & \text { if } t=f\left(t_{1}, \ldots, t_{n}\right) \\
t & \text { if } t \text { is a variable }
\end{array}\|t\|_{l l}= \begin{cases}1+\|X s\|_{l l} & \text { if } t=[X \mid X s] \\
t & \text { if } t \text { is a variable } \\
0 & \text { otherwise }\end{cases}\right.
$$

Note that the norms are symbolic as they map a term to a value that can possibly include variables. An occurrence of a variable $X$ in a symbolic norm means the "size" of $X$ with respect to the given norm.

Example 1.

$$
\begin{array}{ll}
\|f(a, b, g(c))\|_{t s}=5 & \|f(a, b, g(c))\|_{l l}=0 \\
\left\|\left[X_{1}, X_{2}, X_{3}\right]\right\|_{t s}=4+X_{1}+X_{2}+X_{3} & \left\|\left[X_{1}, X_{2}, X_{3}\right]\right\|_{l l}=3 \\
\left\|\left[X_{1}, X_{2} \mid X_{3}\right]\right\|_{t s}=2+X_{1}+X_{2}+X_{3} & \left\|\left[X_{1}, X_{2} \mid X_{3}\right]\right\|_{l l}=2+X_{3}
\end{array}
$$

If a norm maps a term to an expression that does not contain variables, the term is said to be instantiated enough:

Definition 1. (From [13]) A term $t$ is instantiated enough with respect to a symbolic norm $\|$.$\| if \|t\|$ is a ground term.

In Example 1, the term $\left[X_{1}, X_{2}, X_{3}\right]$ is instantiated enough with respect to the listlength norm (since $\left\|\left[X_{1}, X_{2}, X_{3}\right]\right\|_{l l}=3$ ), but not with respect to the termsize norm (since $\left\|\left[X_{1}, X_{2}, X_{3}\right]\right\|_{t s}=4+X_{1}+X_{2}+X_{3}$, the latter expression still containing variables). This characteristic can be used to define the conditions under which a call to a predicate terminates. For example, a call to the wellknown append/3 predicate can be shown to terminate when either its first or its third argument is instantiated enough with respect to the listlength norm.

To the best of our knowledge, the only automatic binding-time analysis for unmoded logic programs that incorporates termination conditions is [3]. It uses 
a Pos-based analysis to compute a safe approximation of what arguments in a predicate call will be instantiated enough during specialisation and combines this information with the termination condition of the called predicate in order to annotate the call as either static or dynamic. The approach is appealing as it separates the actual analysis from the construction of termination conditions, which can be given by the user or automatically derived by a separate termination analysis (like e.g. [15]). However, basing the decision to unfold a call on the termination characteristics of the call imposes considerable restrictions on the unfolding possibilities. The fact that a call is marked static only in case it terminates implies that only calls that can be completely unfolded to true or fail are unfolded. While this approach might be appropriate for some applications, it is not for general logic programs. Consider the following example, implementing a simple Vanilla meta interpreter.

Example 2. Consider the meta interpreter depicted in Fig. 1. The interpreter has the member $/ 2$ and append/3 predicates as object program. The clauses are numbered for later reference.

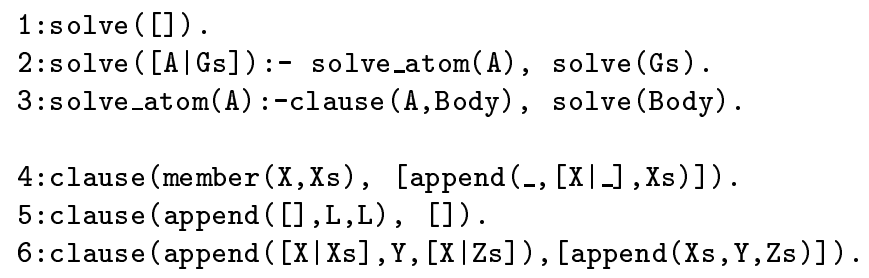

Fig. 1. Vanilla meta interpreter

Assume we want to specialise the meta interpreter from Example 2 with respect to the query solve $([\operatorname{member}(\mathrm{X}, \mathrm{Xs})])$ in order to remove the interpretation overhead and to obtain the object-level definitions of member $/ 2$ and append $/ 3$. Any sensible termination analysis will indicate possible non-termination for this query, the reason being of course that, since an object level call member (X, Xs) does not terminate, neither will the meta call solve ( $[\operatorname{member}(X, X s)])$. Hence, if we take termination of a call as its unfolding condition, no call to solve/1 will be annotated static by the analysis and consequently no such call will be unfolded during specialisation, resulting in a program that is far from optimally specialised.

Intuitively, however, we can see that it is perfectly safe to unfold all calls to the solve/1 predicate as long as the intermediate calls to solve_atom/ 1 are residualised. The idea is that the solve/1 predicate in a sense only performs the parsing of an object goal (deconstructing a list of object atoms), which is terminating in Example 2 and could hence be performed during specialisation. Thus, residualising the calls to solve_atom/2 and unfolding the others results in the specialised program depicted in the left-hand side of Fig. 2. Applying a standard structuring filtering transformation [8] results in the program depicted in the right-hand side which corresponds with (a renaming of) the traditional 
notion of "termination" of program to its termination with respect to the leftto-right selection rule. In what follows, we focus in particular on the concepts and terminology of [4], as this work provides the necessary foundations for our binding-time analysis. Given a symbolic norm $\|$.$\| , we define the abstraction of$ a program with respect to $\|$.$\| as the program that is obtained by replacing each$ term $t$ in the program by $\|t\|$.

Example 3. Consider the append/3 predicate and its abstraction with respect to the list-length norm $\|.\|_{l l}$ in Figure 3.

\begin{tabular}{|c|c|}
\hline append & abstract append/3 w.r.t. $\|\| l l$. \\
\hline $\begin{aligned} \operatorname{append}(X, Y, Z):- & X=[], Y=Z . \\
\operatorname{append}(X, Y, Z):- & X=[E \mid X s], Z=[E \mid Z s], \\
& \operatorname{append}(X s, Y, Z s) .\end{aligned}$ & $\begin{aligned} \operatorname{append}(X, Y, Z):- & X=0, Y=Z . \\
\text { append }(X, Y, Z):- & X=1+X s, Z=1+Z s, \\
& \text { append }(X s, Y, Z s) .\end{aligned}$ \\
\hline
\end{tabular}

Fig. 3. The listlength abstraction of append/3

For simplicity, we assume a normalised representation of clauses in which the head of a clause contains only distinct variables; all unifications are thus explicit in the body of the clause. An abstract program is formally defined over a first order constraint logic programming language denoted $\operatorname{CLP}(\mathcal{N})$. Constraints in $\operatorname{CLP}(\mathcal{N})$ are conjunctions of the relations $\{=, \leq, \geq,<,>\}$ on terms $\mathcal{T}$ constructed from the program's variables and the set of function symbols $\mathbb{N} \cup\{+/ 2\}$. For two atoms $A=p\left(t_{1}, \ldots, t_{n}\right)$ and $B=p\left(t_{1}^{\prime}, \ldots, t_{n}^{\prime}\right)$, we use $A=B$ as an abbreviation for the constraint $\bigwedge_{i=1}^{n}\left(t_{i}=t_{i}^{\prime}\right)$. A clause in $\operatorname{CLP}(\mathcal{N})$ is of the form $H \leftarrow \mu, B_{1}, \ldots, B_{n}$ where $\mu$ is a constraint and $H, B_{1}, \ldots, B_{n}$ are atoms constructed from the program's predicate symbols and $\mathcal{T}$. For a program $P$, we denote its abstraction with respect to the norm $\|$.$\| by P_{\|.\|}$. We assume that each clause in $P_{\|.\|}$has a unique number associated with it and where appropriate, we denote with $i$ : $C$ the clause $C$ with number $i$. Computations in $\operatorname{CLP}(\mathcal{N})$ are performed over $\mathbb{N}$ with the standard interpretations for $\{=, \leq, \geq,<,>\}$.

We follow the approach of [4], and compute a finite approximation of the abstract binary unfoldings semantics of a program $P$. The abstract binary unfoldings semantics of $P$ consists of a (possibly infinite) set of abstract binary clauses. Where the abstract program expresses existing relations on the size of the arguments in the program, the associated abstract binary clauses express relations on the sizes of the arguments in subsequent calls that can occur in $P_{\|.\|}$. We slightly adapt some definitions of [4] to enable the analysis to produce the more detailed information required for our binding-time analysis. A first definition is that of a binary clause, which we generalise to the notion of a labelled abstract binary clause.

Definition 2. A labelled abstract binary clause is a clause in $C L P(\mathcal{N})$ that is either of the form $H \stackrel{i, j}{\leftarrow} \mu$, or $H \stackrel{i, j}{\leftarrow} \mu, B$ where $i, j \in \mathbb{N}$. The set of all such binary clauses is denoted by $\mathcal{B} C$. A clause of the first form, $H \stackrel{i, j}{\leftarrow} \mu$ is also referred to as a labelled constrained atom. 
The set of labelled abstract binary clauses of a program $P_{\|.\|}$is defined as the least fixed point of the operator $T_{P}^{\|\cdot\|}$ defined in Definition 3. The $T_{P}^{\|\cdot \cdot\|}$ operator is adapted from [4] such that it associates a label to each constructed binary clause, referring to how the binary clause was constructed. In what follows, we will often simply refer to "binary clauses" when we mean effectively "abstract labelled binary clauses". Also, we will drop the label from a binary clause when it is unimportant. In Definition $3, \bar{\exists}_{V}\left(\mu_{0}\right)$ denotes the projection of a constraint $\mu_{0}$ onto a set of variables $V$ and $I d$ denotes the set of identity binary clauses, these are clauses of the form $p\left(X_{1}, \ldots, X_{n}\right) \leftarrow p\left(X_{1}, \ldots, X_{n}\right)$. Unfolding an atom with respect to an identity clause results in the atom itself. We furthermore assume that clauses are renamed apart wherever appropriate.

Definition 3. $T_{P}^{\|\cdot\|}: \wp(\mathcal{B} C) \mapsto \wp(\mathcal{B} C)$ is defined as

$$
T_{P}^{\|\cdot\|}(I)=\left\{\begin{array}{l|l}
H \stackrel{i, j}{\leftarrow} \mu, B & \begin{array}{l}
C=i: H \leftarrow \mu_{0}, B_{1}, \ldots, B_{m} \in P_{\|\cdot\|}, 1 \leq j \leq m, \\
\left\langle A_{k} \leftarrow \mu_{k}\right\rangle_{k=1}^{j-1} \in I \\
A_{j} \leftarrow \mu_{j}, B \in I \cup I d, j<m \Rightarrow B \neq t r u e \\
\mu^{\prime}=\mu_{0} \wedge \bigwedge_{k=1}^{j}\left(\mu_{j} \wedge\left\{B_{k}=A_{k}\right\}\right) \\
\mu=\bar{\exists}_{\operatorname{vars}(\langle H, B\rangle)}\left(\mu^{\prime}\right)
\end{array}
\end{array}\right\}
$$

Given a set of binary clauses $I, T_{P}^{\|\cdot\|}(I)$ is a new set of binary clauses constructed by unfolding prefixes of clauses in $P_{\|.\|}$. If $H \leftarrow \mu, B_{1}, \ldots, B_{m}$ is a clause in $P_{\|.\|}$, for each $1 \leq j \leq m$, the body atoms $B_{1}, \ldots, B_{j-1}$ are unfolded with respect to constrained atoms in $I$ and the corresponding instance of $B_{j}$ is unfolded with respect to a binary clause $H_{j} \leftarrow \mu_{j}, B(B \neq$ true $)$ from $I \cup I d$. Note that the use of the identity clause to "unfold" $B_{j}$ results in a binary clause of the form $H \leftarrow \mu, B_{j}$ (which expresses that a call unifying with $\mu, H$ results in a call unifying with $\left.\mu, B_{j}\right)$. Constrained atoms are allowed to unfold $B_{j}$ only in case $j=m$; indeed, an answer is obtained only in case all body atoms are unfolded by a constrained fact. Note that the label associated to a clause constructed by $T_{P}^{\|\| \cdot \|}$ carries information on how the clause was constructed: a clause $H \stackrel{i, j}{\leftarrow} \mu, B$ is created by resolving the $j-1$ leftmost body atoms of the $i$ 'th clause of $P_{\|\cdot\|}$ with constrained atoms and the $j$ 'th atom with a binary clause.

In general, the least fixed point of $T_{P}^{\|\cdot\|}, \operatorname{lfp}\left(T_{P}^{\|\cdot \cdot\|}\right)$ is an infinite set of binary clauses, as illustrated in the next example (from [4]).

Example 4. Reconsider the append/3 predicate from Example 3. The abstract binary unfoldings are computed as in Fig. 4.

To obtain a finitary analysis, different approaches exist to further approximate the abstract domain.

Example 5. Reconsider the abstract binary unfoldings of append/3 from Example 4. Further abstracting using polyhedral approximations [2] (thereby ar- 
(1) $\left(T_{P}^{\|\cdot\|}\right)^{1}(\emptyset)=\left\{\begin{array}{l}\text { append }(X, Y, Z) \stackrel{1,1}{\leftarrow} X=0, Z=Y . \\ \text { append }(X, Y, Z) \stackrel{2,1}{\leftarrow} \\ X=1+X s, Z=1+Z s, \\ \text { append }(X s, Y, Z s) .\end{array}\right\}$
(2) $\left(T_{P}^{\|\cdot\|}\right)^{2}(\emptyset)=\left\{\begin{array}{c}\text { append }(X, Y, Z) \stackrel{2,1}{\leftarrow} X=1, Z=1+Y . \\ \text { append }(X, Y, Z) \stackrel{2,1}{\leftarrow} \\ X=2+X s, Z=2+Z s, \\ \text { append }(X s, Y, Z s) .\end{array}\right.$
(3) $\left(T_{P}^{\|\cdot\|}\right)^{3}(\emptyset)=\left\{\begin{array}{c}\text { append }(X, Y, Z) \stackrel{2,1}{\leftarrow} X=2, Z=2+Y . \\ \text { append }(X, Y, Z) \stackrel{2,1}{\leftarrow} \\ X=3+X s, Z=3+Z s, \\ \text { append }(X s, Y, Z s) .\end{array}\right\} \cup\left(T_{P}^{\|\cdot\|}\right)^{1}(\emptyset)$

Fig. 4. Computing the abstract binary unfoldings.

bitrarily keeping one of the involved labels) results in the set

$$
\operatorname{lfp}\left(T_{P}^{\|\cdot\|}\right)=\left\{\begin{array}{c}
\text { append }(X, Y, Z) \stackrel{1,1}{\leftarrow} Z=Y+X . \\
\text { append }(X, Y, Z) \stackrel{2,1}{\leftarrow} \\
X s<X, Z s<Z, Y s=Y, \\
\text { append }(X s, Y s, Z s) .
\end{array}\right\}
$$

The binary clauses capture size relations that exist between the arguments of subsequent predicate calls. In order to be useful for termination analysis, these size relations must be combined with instantiation information, that specifies which of the arguments are instantiated enough with respect to the norm under consideration. Such instantiation information is obtained by a standard groundness analysis on the abstracted program. In what follows we consider, as in [4], an abstract domain that combines size relations and instantiation information. We denote with $\mathrm{mgu}^{\alpha}$ the abstract most general unifier over this domain, and denote with $\approx$ the equivalence of syntactic objects. For a program $P$, we denote with $\mathcal{B}_{P}$ the finite set of abstract binary clauses which approximates, with respect to some given abstraction, $\operatorname{lfp}\left(T_{P}^{\|\cdot\|}\right)$ over the combined abstract domain.

In termination analysis, one is interested in the termination behaviour of a specific call with respect to the given program. First, we define the abstraction of a call $p\left(t_{1}, \ldots, t_{n}\right)$ with respect to a norm $\|\cdot\|$ as $\left\|p\left(t_{1}, \ldots, t_{n}\right)\right\|=$ $p\left(\left\|t_{1}\right\|, \ldots,\left\|t_{n}\right\|\right)$. The (possibly infinite) set of calls that arise during computation of an initial call $Q$ in $P$ can be approximated by a finite set of abstract calls, $\operatorname{call}_{P}^{\alpha}(Q)$, which is determined as follows:

Definition 4. Given a program $P$, an abstract initial call $Q$ and a finite set of abstract binary clauses $\mathcal{B}_{P}$ approximating lfp $\left(T_{P}^{\|\cdot\|}\right)$.

$$
\operatorname{calls}_{P}^{\alpha}(Q)=\left\{B \theta \mid \begin{array}{l}
H \leftarrow B \in \mathcal{B}_{P}, \\
\theta=m g u^{\alpha}(Q, H)
\end{array}\right\}
$$


The work of [4] defines a sufficient condition to show termination of a call $Q$ with respect to a program $P$. Given a finite approximation $\mathcal{B}_{P}$ of $\operatorname{lfp}\left(T_{P}^{\|\cdot\|}\right)$, it is sufficient to show for each call $C \in \operatorname{calls}_{P}^{\alpha}(Q)$ a strict decrease in size from the head to the single body atom for all recursive clauses of $\mathcal{B}_{P}$ that unify with the call $C$. Adding labels to the abstract binary clauses enables one to reformulate the termination condition of [4] at the level of the original clauses of $P$. To that end, we introduce the notion of a clause being loop safe in one of its body atoms. Intuitively, if we say that a clause $H \leftarrow B_{1}, \ldots, B_{n}$ is loop safe in its $i$ 'th body atom with respect to a set of (abstract) calls $S$, this means that none of the calls in $S$ unifying with $H$ will spawn an infinite derivation through (instances of) this body atom.

Definition 5. Given a program $P$, a set of abstract calls $S$ and a finite approximation $\mathcal{B}_{P}$ of lfp $\left(T_{P}^{\|\cdot\| \|}\right)$. Assume there exist a binary clause $\beta=H \stackrel{i, j}{\leftarrow} \mu, B \in \mathcal{B}_{P}$ and a call $C \in S$ such that $\theta=m g u^{\alpha}(H, C)$ and $B \theta \approx C$. Let $i_{1}, \ldots, i_{k}$ be the argument positions that are instantiated enough both in $H \theta$ and $B \theta$. We denote these arguments by $(H \theta)_{i_{1}}, \ldots,(H \theta)_{i_{k}}$ and $(B \theta)_{i_{1}}, \ldots,(B \theta)_{i_{k}}$. We say that the clause $i$ of $P$ is loop safe with respect to $S$ in body atom $j$ if for each such $\beta \in \mathcal{B}_{P}$ and $C \in S$ there exists a function $f$ such that

$$
\mu=f\left((H \theta)_{i_{1}}, \ldots,(H \theta)_{i_{k}}\right)>f\left((B \theta)_{i_{1}}, \ldots,(B \theta)_{i_{k}}\right) .
$$

Note that Definition 5 takes only those (abstract) calls and binary clauses into account such that applying the most general unifier of the head of the clause and the call on the body atom results in a recursive call with an equivalent call pattern. See [4] for details on why this is sufficient. Given Definition 5 from above, we define for a clause $i$ of $P$ and a set of abstract calls $S$,

$$
L S_{P, S}^{i}=\{j \mid \text { the clause } i \text { is loop safe w.r.t. } S \text { in body atom } j\}
$$

Example 6. Let $P$ denote the program consisting of the append/3 predicate with the abstract binary unfoldings from Example 5 and let $Q$ denote the abstracted initial call append $(0, Y, Z)$. This call unifies (through $\left.\mathrm{mgu}^{\alpha}\right)$ only with the head of the binary clause labelled $(1,1)$, since unification of append $(0, \mathrm{Y}, \mathrm{Z})$ with the other clause, labelled $(2,1)$, fails due to the fact that the size constraints are not satisfied since no $X s<0$. Hence, we have that both clauses of append $/ 3$ are loop safe with respect to $S=\operatorname{calls}_{P}^{\alpha}(\operatorname{append}(0, Y, Z))=\{\operatorname{append}(0, Y, Z)\}$. Or, we have that $l s_{P, S}^{1}=\emptyset$ and $L S_{P, S}^{2}=\{1\}$.

An important result from [4] can now be reformulated as follows: Given a program $P$ and initial goal $Q$, if each clause of $P$ is loop safe with respect tot calls $_{P}^{\alpha}(Q)$ in each of its body atoms, then $Q$ terminates with respect to $P$.

\subsection{From Termination Analysis to Binding-time Analysis}

Recall that we want to enable the termination analysis to indicate why termination of a program cannot be proven. We therefore define the notion of the leftmost possibly looping atom of a clause as the leftmost atom of the clause for which the termination analysis cannot prove that it is loop safe. 
Definition 6. Consider a program $P$, a set of abstract calls $S$ and a finite approximation $\mathcal{B}_{P}$ of lfp $\left(T_{P}^{\|\cdot\|}\right)$. Let $H \leftarrow B_{1}, \ldots, B_{n}$ be the $i$ 'th clause of $P$. We define its leftmost possibly looping body atom as follows

$$
L L A_{P, S}^{i}= \begin{cases}\min \left(\{1, \ldots, n\} \backslash L S_{P, S}^{i}\right) & \text { if }\left(\{1, \ldots, n\} \backslash L S_{P, S}^{i}\right) \neq \emptyset \\ \text { undefined } & \text { otherwise }\end{cases}
$$

Example \%. Let $P$ denote the program consisting of the append/3 predicate with the abstract binary unfoldings from Example 5 and let $Q$ denote the abstracted initial call append $(\mathrm{X}, 0, \mathrm{Z})$ and $S=\operatorname{calls}_{P}^{\alpha}(Q)$. Although we still have that $L S_{P, S}^{1}=\emptyset$, we also have that $L S_{P, S}^{2}=\emptyset$. Indeed, the second clause in the append program is not loop safe with respect to $S$ in its only body atom, due to the existence of the binary clause labelled $(2,1)$ (see Example 5). Unifying this clause with the call append $(\mathrm{X}, 0, \mathrm{Z})$ results in the binary clause

$$
\operatorname{append}(X, 0, Z) \leftarrow \operatorname{append}(X s, 0, Z s) .
$$

Only the second argument of both atoms is instantiated enough, and there does not exist a function $f$ such that $X>X s, Z>Z s \models f(0)>f(0)$. Hence, we have that $L L A_{P, S}^{2}=1$.

In general, finding the leftmost looping atom of a clause (if it exists) is an undecidable problem. In practical systems, however, the function $f$ in Definition 5 is fixed, and is usually defined as a linear combination of the involved arguments. When $f$ is fixed, the test of Definition 5 can be evaluated, and consequently the sets $L S_{P, Q}^{i}$ and $L L A_{P, Q}^{i}$ can be computed. For any clause $i$ in $P, L L A_{P, S}^{i}$ provides a safe approximation of the leftmost looping atom in the clause, since it is guaranteed that no atom to its left can be looping, when the program is evaluated with respect to an initial call $Q$ and $S=\operatorname{calls}_{P}^{\alpha}(Q)$.

Now, we have developed the necessary machinery to define the binding-time analysis, which requires annotating the atoms in each of the program's clauses. Annotating the body atoms of a clause usually consists of adding a label to each atom, specifying whether the atom is unfolded during specialisation, or residualised. In this work, however, we focus on the termination aspects of the unfolding, and hence employ a slightly different notion of annotations. In what follows, we simply replace atoms that should be residualised by true. Doing so permits one to study the termination behaviour of a specialiser that simply unfolds the static atoms (and generates code for those that are residualised) by studying the termination behaviour of the annotated program under normal evaluation.

Definition 7. Given a clause $H \leftarrow B_{1}, \ldots, B_{n}$, an annotated version of the clause is a clause $H \leftarrow B_{1}^{\prime}, \ldots, B_{n}^{\prime}$, where for each $i$ such that $1 \leq i \leq n$, it holds that either $B_{i}^{\prime}=B_{i}$ or $B_{i}^{\prime}=$ true. An annotated version of a program $P=\bigcup_{i} C_{i}$ is a program $P^{\prime}=\bigcup_{i} C_{i}^{\prime}$ such that for every such clause $C_{i}$, it holds that $C_{i}^{\prime}$ is an annotated version of $C_{i}$. 
Note that, according to Definition 7, every clause is an annotated version of itself. When annotating a program, one generally wants to mark as many atoms static as possible, while guaranteeing termination of the unfolding. This is the main idea behind the analysis presented in this work. Suppose we have to annotate a program $P$ with respect to an initial call $Q$. If we can prove that $Q$ terminates with respect to $P$, the annotated version of $P$ is simply $P$ itself (every atom is annotated static). Hence, during specialisation, the goal $Q$ will be completely unfolded, and specialisation of $Q$ boils down to plain evaluation of $Q$ in $P$. If, on the other hand, termination of $Q$ with respect to $P$ can not be proven by our analysis, at least one of $P$ 's clauses must have a leftmost looping atom, and we mark this atom dynamic (by replacing it by true in $P$ ). This process is repeated until the annotated program is loop safe. This is the main intuition behind the algorithm for binding-time analysis which is depicted in Fig. 5. Note

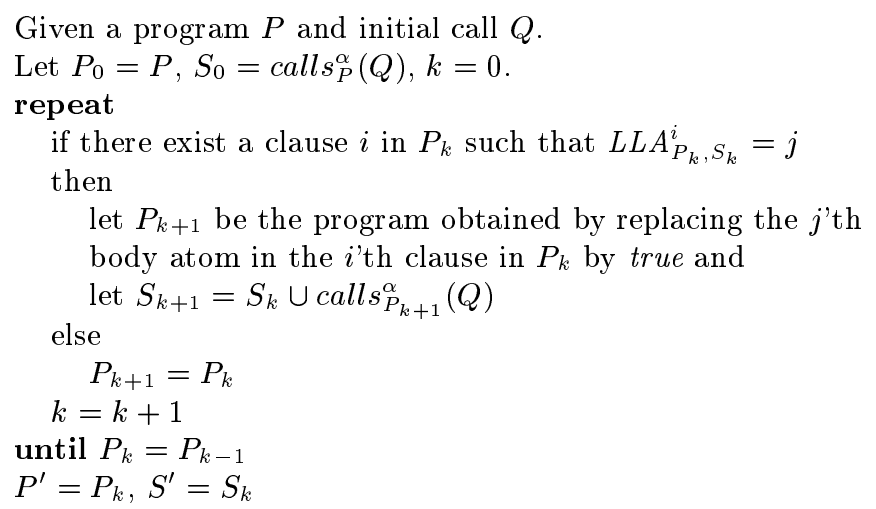

Fig. 5. The BTA algorithm.

that the algorithm is non deterministic: if several clauses $i$ exist in $P_{k}$ for which $L L A_{P_{k}, S_{k}}^{i}$ is defined, one of these atoms must be selected for replacement by true. Also note the construction of the set $S^{\prime}$ : starting from the program's initial abstract callset $S_{0}$, the abstract callset of the annotated program is added in each round of the algorithm. Doing so guarantees that the calls that are unfolded are correctly represented by an abstract call in $S^{\prime}$, but it also ensures that $S^{\prime}$ contains abstractions of the (concrete instances of the) calls that were replaced by true during the process. In other words, the set $S^{\prime}$ contains an abstraction of every call that is encountered (unfolded or residualised) during specialisation of $P$ with respect to the initial call $Q$.

Termination of the BTA algorithm is straightforward, since in every iteration an atom in a clause is replaced by true, and the program only has a finite number of atoms. The result of the algorithm includes a logic program $P^{\prime}$ with respect to which evaluation of any call that is abstracted by a call in the set $S^{\prime}$ terminates. This is an important result, as it implies termination of specialisation of such a call when the specialisation is performed by following the annotations corresponding to $P^{\prime}$. More formally, we can define such an unfolding rule as follows: 
Definition 8. An atom $A$ in a goal at the leaf of an SLD-tree is selectable unless it is an instance of an atom in $P$ that is replaced by true in $P^{\prime}$. The unfolding rule $U_{b t a}$ unfolds the leftmost selectable atom in each goal of the SLD-tree under construction. If no atom is selectable, no further unfolding is performed.

Now, the SLD-tree built by $U_{b t a}$ for $P \cup\{Q\}$ is finite since $U_{b t a}$ unfolds - apart from $Q$ - only atoms that are instances of an atom in $P^{\prime}$. Moreover, every atom $A$ that is not unfolded by $U_{b t a}$ - and hence present in the residual program - is a concretisation of a call in $S^{\prime}$. Hence, building an SLD-tree for $P \cup\{A\}$ again terminates. This kind of termination is often referred to as local termination: building a finite SLD-tree for each atom that is specialised. Constructing a finite set of atoms that are specialised involves a second kind of termination, often referred to as global termination. Global termination is not guaranteed by BTA alone. Indeed, the abstract callset $S^{\prime}$ is a finite set, but an infinite number of concretisations of the calls in $S^{\prime}$ may be constructed during specialisation.

\section{Experimental Evaluation}

Table 1 summarises a number of experiments that were run with an implementation of the described binding-time analysis. The second column (Round1) presents the timings for termination analysis of the original program (in which all calls are annotated static). In case the outcome of the analysis is possible non-termination, the third column (Round2) presents the timings for termination analysis of the program in which the problematic call is annotated dynamic. None of the benchmarks, which are taken from the DPPD library [12], required more than two rounds of the termination analysis to derive a terminating annotated program. The benchmarks were run under SICStus Prolog 3.7.1 on a Sun Ultra E450 server with $256 \mathrm{Mb}$ RAM operating under SunOS 5.6. The fourth

\begin{tabular}{|l|c|c|c|c|c|}
\hline \multicolumn{1}{|c|}{ Benchmark } & Round 1 & Round 2 & LOGEN & Total & MIXTUS \\
\hline ex_depth & 240.0 & 230.0 & 4.4 & 474 & 200 \\
match & 470.0 & 180.0 & 2.4 & 652 & 50 \\
map.rev/reduce & 200.0 & - & 4.3 & 204 & 100 \\
parser & 100.0 & 50.0 & - & 150 & - \\
regexp1-3 & 740.0 & 280.0 & 15.1 & 1035 & 670 \\
transpose & 210.0 & 150.0 & 7.0 & 367 & 290 \\
\hline
\end{tabular}

Table 1. Timings (in ms) for the binding-time analysis and full specialisation.

column in Table 1 contains the time needed to produce the specialised program using the LOGEN system [9]. The final column contains the specialisation time of Mixtus [17] - a well-known on-line specialiser for Prolog - as a reference point. Table 1 shows that binding-time analysis is the most expensive operation in the specialisation process. However, recall that the results of binding-time analysis can be used to perform several specialisations (with respect to values approximated by the binding-times from the partial deduction query). For the considered benchmarks, the cost of binding-time analysis will be recovered after 
a few specialisations compared with Mixtus. Typical speedups obtained by the specialisation range from 1.15 to 2.23 for these benchmarks.

\section{Discussion}

In this work, we have taken a rather unusual approach towards binding-time analysis. Well-known techniques from termination analysis are adapted and used to annotate a program in successive steps, until it can be proven that specialisation - rather than full evaluation - of a call terminates. Preliminary experiments show that the approach is feasible and results in more liberal unfoldings than with earlier known approaches that use separately generated (or hand crafted) termination conditions like [3]. Examples are the solve example and the regexp benchmark from above, since in these programs, the ability to partially unfold a predicate call is crucial to achieve a fair amount of specialisation. In the solve example from Section 2, our analysis is able to compute that the call to solve_atom is the leftmost looping atom in the program's second clause and that annotating this call dynamic suffices to obtain termination, and hence the results devised in Section 2. The regexp benchmark is depicted in Fig. 6. Assume we want to specialise this program for a query in which the first argument (the regular expression that needs to be matched) is ground. Termination is not guaranteed, hence a binding-time analysis based on termination conditions would not be able to unfold any call to gen/3. Still, our binding-time analysis is able to spot the only problematic call sequence from head to body atom (underlined in Fig. 6), marking the other calls static such that all calls except for the underlined one can be unfolded during specialisation.

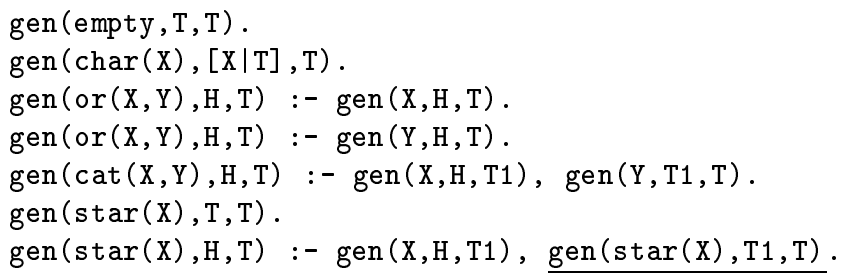

Fig. 6. The regexp benchmark

In contrast with [3] - being a polyvariant analysis - our binding-time analysis is monovariant: it creates only a single annotated version of every predicate. This is not an issue in the benchmarks presented above, but for more involved programs, the resulting annotations are likely to be suboptimal, since calls in which different arguments are instantiated enough are likely to expose a different termination behaviour, and hence they might profit from being unfolded differently. Another characteristic of the analysis that might be an issue when analysing larger programs, is that the binding-time analysis basically deals with boolean binding-times: either a value is instantiated enough with respect to a norm, or it is not. Although some work exists towards automating the process of choosing a suitable norm, most systems require the norm to be selected by the user. 
The particular norm that is used by the system determines the granularity of a term that is considered static for binding-time analysis. The use of the termsize norm, for example, corresponds to distinguishing, during binding-time analysis, between definitely ground terms and possibly non ground terms. The use of the listlength norm, on the other hand, enables one to consider a term static when it is instantiated up to a list skeleton. Even if the norm is provided manually, finding a suitable norm might not be trivial or might even be impossible - in particular for programs that employ values of different types. These issues are not due to the binding-time analysis itself, but are rather connected with the termination analysis. We expect better (more precise) termination analyses to lead to better (more precise) binding-time analysis.

In this work, we have used termination analysis to ensure - in an off-line setting - local termination of the specialisation process. A topic for further research is the use of termination analysis towards globally controlling the specialisation process as well. A possible approach towards ensuring (global) termination in offline partial evaluation of functional programs is presented in [1]. In this work, the output of a termination analysis is used to make enough values dynamic such that the program enters - during specialisation - only a finite number of different configurations (where a configuration is defined as a program point together with values for the variables at that program point). If this is the case, the program is said to quasi-terminate and termination of partial evaluation is ensured by memoizing the configurations. A weakness of the approach of [1] is that its termination analysis only recognises "in-situ" decreases, i.e. a decrease in the size of a single argument between recursive calls. A more general termination analysis is developed in [10], capable of dealing with indirect function calls and permuted arguments (lifting the in-situ criterion). Developing an analogous analysis for binding-time analysis is mentioned as an important issue in [10]. The notion of quasi-termination for logic programs has also been explored [6] in the context of termination analysis of tabled logic programs. In [3], it is noted that global termination of the process is ensured if quasi-termination of the program with the residualised predicates tabulated can be established. Precisely how to integrate such a technique with a suitable and refined abstraction mechanism is an interesting topic for further research.

\section{Acknowledgements}

We thank Michael Codish for stimulating discussions and for making the source code of the TerminWeb termination analyser publically available, Michael Leuschel for his interest and for performing some tests with LOGEN and the result of BTA, and anonymous referees for their valuable comments.

\section{References}

1. Peter Holst Andersen and Carsten Kehler Holst. Termination analysis for offline partial evaluation of a higher order functional language. In Proceedings of the Third International Static Analysis Symposium (SAS), pages 67 - 82, 1996. 
2. F. Benoy and A. King. Inferring argument size relationships with CLP(R). In Proceedings of LOPSTR'96, volume 1207 of Lecture Notes in Computer Science, pages 204-223, 1997.

3. Maurice Bruynooghe, Michael Leuschel, and Kostis Sagonas. A polyvariant binding-time analysis for off-line partial deduction. In C. Hankin, editor, Programming Languages and Systems, Proc. of ESOP'98, part of ETAPS'98, pages 27-41, Lisbon, Portugal, 1998. Springer-Verlag. LNCS 1381.

4. M. Codish and C. Taboch. A semantic basis for the termination analysis of logic programs. Journal of Logic Programming, 41(1):103-123, 1999.

5. D. De Schreye and S. Decorte. Termination of logic programs: the never-ending story. Journal of Logic Programming, 19/20:199-260, 1994.

6. S. Decorte, D. De Schreye, M. Leuschel, and B. Martens. Termination analysis for tabled logic programming. In N. Fuchs, editor, Proceedings of LOPSTR'97, number 1463 in Lecture Notes in Computer Science, pages 107-123, 1998.

7. S. Decorte and D. De Schreye. Termination analysis: Some practical properties of the norm and level mapping space. In Joxan Jaffar, editor, Proceedings of the 1998 Joint International Conference and Symposium on Logic Programming (JICSLP98), pages 235-249, Cambridge, 1998. MIT Press.

8. J. Gallagher and M. Bruynooghe. Some low-level source transformations for logic programs. In M. Bruynooghe, editor, Proceedings Meta'90, pages 229-244, Leuven, 1990.

9. J. Jørgensen and M. Leuschel. Efficiently generating efficient generating extensions in Prolog. In O. Danvy, R. Glück, and P. Thiemann, editors, Proceedings Dagstuhl Seminar on Partial Evaluation, pages 238-262, Schloss Dagstuhl, Germany, 1996. Springer-Verlag, LNCS 1110.

10. Chin Soon Lee, Neil D. Jones, and Amir M. Ben-Amram. The size-change principle for program termination. In ACM Symposium on Principles of Programming Languages, volume 28, pages 81-92. ACM press, 2001.

11. M. Leuschel, B. Martens, and D. De Schreye. Controlling generalisation and polyvariance in partial deduction of normal logic programs. ACM Transactions on Programming Languages and Systems, 20(1):208 - 258, 1998.

12. Michael Leuschel. The ECCE partial deduction system and the DPPD library of benchmarks. Obtainable via http://www.cs.kuleuven.ac.be/ 1 pai, 1996.

13. Naomi Lindenstrauss and Yehoshua Sagiv. Automatic termination analysis of logic programs. In Lee Naish, editor, Proceedings of the 14th International Conference on Logic Programming, pages 63-77, Cambridge, 1997. MIT Press.

14. J. W. Lloyd and J. C. Shepherdson. Partial evaluation in logic programming. Journal of Logic Programming, 11(3\&4):217-242, 1991.

15. Fred Mesnard. Inferring left-terminating classes of queries for constraint logic programs. In Michael Maher, editor, Proceedings of the 1996 Joint International Conference and Symposium on Logic Programming, pages 7-21, Cambridge, 1996. MIT Press.

16. T. Mogensen and A. Bondorf. Logimix: A self-applicable partial evaluator for Prolog. In K.-K. Lau and T. Clement, editors, Proceedings LOPSTR'92, pages 214-227. Springer-Verlag, Workshops in Computing Series, 1993.

17. D. Sahlin. Mixtus: An automatic partial evaluator for full Prolog. New Generation Computing, 12(1):7-51, 1993.

18. W. Vanhoof and M. Bruynooghe. Binding-time analysis for Mercury. In D. De Schreye, editor, 16th International Conference on Logic Programming, pages 500 - 514. MIT Press, 1999. 